\title{
Germanica
}

\section{„Marcellus non papa ex cathedra“ Zwei Begegnungen zwischen Hans Blumenberg und Marcel Reich-Ranicki}

Dorit KRUSCHE : "Marcellus non papa ex cathedra ». Deux rencontres entre

Hans Blumenberg et Marcel Reich-Ranicki

Dorit KRUSCHE: "Marcellus non papa ex cathedra". Two Encounters between

Hans Blumenberg and Marcel Reich-Ranicki

Dorit Krusche

\section{OpenEdition}

Journals

Édition électronique

URL : https://journals.openedition.org/germanica/7626

DOI : 10.4000/germanica.7626

ISSN : 2107-0784

\section{Éditeur}

Université de Lille

\section{Édition imprimée}

Date de publication : 1 décembre 2019

Pagination : 65-78

ISBN : 978-2-913857-44-5

ISSN : 0984-2632

\section{Référence électronique}

Dorit Krusche, ${ }_{n, m}$ Marcellus non papa ex cathedra" Zwei Begegnungen zwischen Hans Blumenberg und Marcel Reich-Ranicki", Germanica [Online], 65 | 2019, Online erschienen am: 01 Januar 2022, abgerufen am 07 Januar 2022. URL: http://journals.openedition.org/germanica/7626 ; DOI: https://doi.org/ $10.4000 /$ germanica.7626 


\title{
„Marcellus non papa ex cathedra“6 Zwei Begegnungen zwischen Hans Blumenberg und Marcel Reich-Ranicki
}

\author{
Dorit Krusche \\ Deutsches Literaturarchiv Marbach
}

\section{Von Bloch zu Hegel}

In Schiffbruch mit Zuschauer treffen Goethe und Hegel im Werk von Hans Blumenberg für einen kurzen Moment zusammen. Blumenberg versucht hier zu zeigen, dass die von Goethe zur Schau gestellte unterkühlte Zuschauerposition angesichts der Schlacht bei Jena mühsame, in klassische Form gepresste Beherrschung verrät, während Hegel in Die Vernunft in der Geschichte das Schicksal des Einzelnen in purer Abstraktion einem höheren Ziel unterstellt und den Geschichtsphilosophen als persönlich unbeteiligten Zuschauer idealisiert $^{1}$. Aber was haben Goethe und Hegel mit Hans Blumenberg und Marcel Reich-Ranicki zu tun?

Unterschiedlichere Charaktere als Reich-Ranicki und Blumenberg lassen sich kaum denken. Und doch gibt es auch auf den ersten Blick Gemeinsames: Sie waren Altersgenossen, hatten Sinn und Interesse für

1. - Hans Blumenberg, Schiffbruch mit Zuschauer. Paradigma einer Daseinsmetapher, Frankfurt a.M., Suhrkamp-Verlag, 2000, S. $57 f$. 
Literatur, waren Atheisten und stammten aus bürgerlichen Familien mit jüdischem Hintergrund. Sie lasen und schrieben gern und viel für Zeitungen, waren radikal und empfindlich, Provokateure. Die Konfrontationen zwischen dem Kritiker und dem Philosophen, die hier nachgezeichnet werden sollen, werfen ein bezeichnendes Licht auf die Selbstbilder und ästhetischen Positionen beider.

Über persönliche Begegnungen kann nur spekuliert werden. Schriftlich haben sich ihre Wege und Schwerter jedoch mindestens zwei Mal gekreuzt:

Hans Blumenberg war ein interessierter und kommentierender Zeitungsleser; seine bevorzugten Tageszeitungen waren die Frankfurter Allgemeine Zeitung (FAZ) und die Neue Zürcher Zeitung (NZZ), zu deren Redakteuren er später auch in persönlichem Kontakt stand: Bis zu seinem Lebensende korrespondierte und telefonierte er mit Henning Ritter und Martin Meyer. Was er in Zeitungen gelesen hatte, wurde ausgeschnitten, auf Karteikarten geklebt, diktiert, erörtert.

Am 24. August 1985 las Hans Blumenberg eine Besprechung der von Karola Bloch herausgegebenen Briefe Ernst Blochs aus den Jahren 1903-1975. Geschrieben hatte sie der in Australien lehrende Germanist Gerhard Schulz, den Reich-Ranicki schon vor seinem Eintritt in die FAZ-Redaktion als Autor für das Blatt gewonnen hatte. Schulz rezensierte Blochs Briefe mit viel Ironie und aus großer Distanz, kritisierte vor allem dessen frühes Pathos, seine bis zur Realitätsverleugnung gehende „Werkbesessenheit“2.

Die umstrittene Rezension, die sich im Nachlass Blumenbergs erhalten hat, trägt zwei mit Filzstift ausgeführte Lesespuren. Er strich ein ironisches Zitat des Rezensenten an: „Eine jubelnde Schülerschar. Die Philosophie bricht durch"3. Ein rotes Fragezeichen malte Blumenberg neben den Satz von Bloch: „Anlässlich der Heirat mit der Tochter eines baltischen Brauereibesitzers heißt es: ,Jetzt spielt das Bier bei Hegel' - der gern trank - ,wie auch bei mir in die Philosophie hinein.“" "4. Was bedeutet das Fragezeichen? Blumenberg erklärt das in seinem Leserbrief, den er am 25. Oktober 1985 an Marcel Reich-Ranicki als zuständigen Literaturredakteur schickte. Die Verbindung von Bier und Philosophie bei Hegel und Bloch, um die es in Blochs Brief an Georg Lukács vom 20. Februar 1913 geht, sei keineswegs durch beider Alkoholkonsum, sondern

2. - Gerhard Schulz, „Ein Imperator im Reiche des Geistes. Ernst Blochs Briefe aus den Jahren 1903 bis $1975^{\prime \prime}, F A Z$ vom 24.8.1985.

3. - Ebd.

4. - Der Zeitungsausschnitt findet sich im Nachlass von Hans Blumenberg im Deutschen Literaturarchiv Marbach (DLA). Für die freundliche Genehmigung, aus den Briefen von Hans Blumenberg, Marcel Reich-Ranicki und Siegfried Unseld zu zitieren, danke ich Bettina Blumenberg, Thomas Anz und der Siegfried-UnseldFamilienstiftung, vertreten durch Raimund Fellinger, herzlich. 
durch die Ehen mit Töchtern aus Brauereifamilien gestiftet worden ${ }^{5}$. Blumenberg fährt fort: „Etwas mehr Hintergründigkeit darf man schon voraussetzen, wenn Hegelianer einander Briefe schreiben, und Bloch wußte genauer, was er sagte, und konnte sich darauf verlassen, daß es verstanden würde"6. Auch der Kommentar des Briefbandes verweise nur darauf, dass in „Hegels Briefen häufig von Bier und Wein die Rede“" sei”.

Blumenbergs Glosse, die später unter dem Titel „Wie Hegel vom Schnupfer zum Trinker wurde" in Die Verführbarkeit des Philosophen ${ }^{8}$ aus dem Nachlass erschien, liest sich trotz seines Ärgers locker und launig. Kaum ist dem kurzen Text anzumerken, dass es dem Rezensenten des Rezensenten um mehr als um eine Korrektur ging. Im Plauderton demontiert Blumenberg darin nicht nur die Besprechung von Gerhard Schulz, sondern auch den Kommentar der Briefausgabe selbst. Was machte es für Blumenberg die Mühe wert, eine Glosse zu einem solchen Thema zu schreiben? Wäre es ihm um philologische Genauigkeit gegangen, hätte er dem Rezensenten zuerst den kleinen Schreibfehler - „hinein“ anstelle von „,herein“ - ankreiden können, den er kommentarlos in seinem Zitat berichtigt hat.

Marcel Reich-Ranicki antwortete wenig später, er habe Blumenbergs Leserbrief „mit großem Interesse und mit Vergnügen“ gelesen und kontert: „Wie schade, daß wir Ihre erhellenden und überzeugenden Darlegungen nicht drucken können, denn nach alten Regeln dieses Hauses müssen Leserbriefe, die wir bringen, sich auf Artikel beziehen, die im Laufe der letzten vier Wochen gedruckt wurden [...]"9. Er gebe den Brief aber an den Autor der Rezension weiter. Für den Redakteur war der Fall damit erledigt.

Blumenberg sah sich unverhofft auf die Zuschauerbank verwiesen. Reich-Ranickis Zugriff auf das Medium Zeitung hatte sich nonchalant gegen seinen Anspruch auf wissenschaftliche Redlichkeit durchgesetzt.

5. — Georg Wilhelm Friedrich Hegel und Marie von Tucher (1791-1855), deren Patrizierfamilie allerdings erst 1855 eine Brauerei übernahm, heirateten 1811. Das Bier kam eigentlich erst durch die Heirat von Hegels Sohn Karl mit seiner Cousine Susanna Maria von Tucher in die Familie. Ernst Blochs erste Ehefrau, die Bildhauerin Else von Stritzky (1883-1921), stammte aus einer wohlhabenden baltischen Brauereifamilie.

6. - Leserbrief von Hans Blumenberg vom 25.10.1985, Nachlass Hans Blumenberg, DLA Marbach, später gedruckt als „Wie Hegel vom Schnupfer zum Trinker wurde“, in: Hans Blumenberg, Die Verführbarkeit des Philosophen, Frankfurt a.M., Suhrkamp-Verlag, 2000, S. 49.

7. - Ebd. Die Bemerkung findet sich in: Ernst Bloch, Briefe aus den Jahren 1903-1975, hrsg. von Karola Bloch und Uwe Opolka, Bd. 1, Frankfurt a. M., SuhrkampVerlag, 1985, S. 103.

8. - Hans Blumenberg, Die Verführbarkeit des Philosophen, a.a.O., S. 49.

9. - Nachlass Hans Blumenberg, DLA Marbach. Ob Reich-Ranicki zu diesem Zeitpunkt wusste, wer der von ihm als „Dr. Blumenberg“ angesprochene PhilosophieProfessor eigentlich war, ist nicht ganz sicher. 
In den Paradigmen zu einer Metaphorologie hatte Blumenberg 1960 geschrieben: ,[E]rkennen und ,veröffentlichen“ ist für den professionell fungierenden Forscher der Neuzeit so gut wie dasselbe"10. Aber wie durchsetzungsfähig ist ein solches Postulat, wenn der Erkenntnis die Öffentlichkeit aus fadenscheinig formalen Gründen verweigert werden kann?

Hans Blumenberg wendet sich am 29. November 1985 an seinen Verleger und Freund Siegfried Unseld. Dem Verleger gegenüber akzentuiert Blumenberg nun eher den Fehler in der Briefausgabe seines Verlages, wenn er von der ,durch den Kommentar bewirkten Fehlleistung des Rezensenten" 11 spricht. Er legt den Leserbrief bei, aus dem er eine Glosse mit dem Titel „Wie Hegel vom Schnupfer zum Trinker verkam" machen möchte, fragt nach eventuellen Rücksichten bei einer geplanten Veröffentlichung: „Es ist ein Witz, daß ein dezidierter Verächter Hegels das machen würde, und das wieder vergnügt mich" 12 . Siegfried Unseld, der seinen Autor und dessen polemischen Sinn gut kennt, überhört den an ihn gerichteten Vorwurf. Er versucht positiv auszuweichen, bagatellisiert die Angelegenheit und antwortet am 16. Dezember 1985 in aller Kürze, dass er nichts gegen die Veröffentlichung der Glosse einzuwenden habe: „Köstlich! Aber wer kennt schon die verwandtschaftlichen Beziehungen der Frauen von Philosophen"13?

In Blumenbergs Briefkonvolut liegt der Entwurf zu einem Brief oder Essay, von dem nicht zu sagen ist, ob ihn der Adressat Siegfried Unseld - egal in welcher Form - jemals zu Gesicht bekommen hat. Als Motto oder Überschrift hat Blumenberg nachträglich über den Text gesetzt: „Tenor der Br[ie]fe., die man an Pedanten schreibt: Nun weiß er sogar darüber was"14. Der darauf folgende Text ist schon deshalb besonders aufschlussreich, weil Hans Blumenberg hier etwas tut, was man ansonsten selten bei ihm findet. Er erläutert und interpretiert seinen eigenen Text, legt in der dritten Person Singular Hintergedanken und Beweggründe offen:

Der Adressat der rhetor[ischen] Frage [gemeint ist der Autor H.B. selbst, D.K.], weiß aber auch nichts (!) über verwandtsch.[aftliche] Bez. [iehungen] v.[on] $\mathrm{Ph}$.[ilosophen]fr.[auen]. Er weiß nur, wann es an der Zeit ist, sich über einen solchen Fall um jeden Preis und mit allen Mitteln zu informieren. Und er will, indem er diese Fälligkeit an den Tag bringt, nicht die neue Unwissenheit der Komm. [entatoren] anprangern, sondern

10. - Hans Blumenberg, Paradigmen zu einer Metaphorologie, Frankfurt a. M., Suhrkamp-Verlag, 1998, S. 70.

11. - Nachlass Hans Blumenberg, DLA Marbach.

12. - Ebd.

13. - Ebd.

14. - Nachlass Hans Blumenberg, DLA Marbach. 
ihre Unfähigkeit zu erkennen, wann sie sich über was zugunsten ihrer Aufgabe ins Bild setzen müssen. Der Vorgang „Bloch/Hegel“ beginnt nicht mit dem Wissen über Verwandtschaftsverh.[ältnisse] v.[on] Philosophenfrauen, die mich so wenig interessieren, wie wahrsch. [einlich] SU [Siegfried Unseld].

Der Vorgang beginnt damit, daß ein Rezensent in Melbourne die beiden Bände der Bloch-Korr.[espondenz] f.[ür] d.[ie] FAZ zu rez[ensieren] bek[ommt], der f[ür] d[ie]se Rez[ension] die Vorraussetz[un]gen nicht besitzt ${ }^{15}$. Aber was er besitzt, ist ein Sensorium f[ür] e[inen] schönen $\&$ bedeutsamen Satz. Er zitiert ihn \& erläutert ihn parenthetisch falsch, weil er dem Kommentator geglaubt hatte. Mußte er dazu schon etwas über das Haus Tucher wissen? Nein. Er mußte nur merken, daß der schöne Satz s[eine] Bedeutung aus e.[iner] vollk.[ommenen] Symmetrie bezieht \& sonst gar nichts wert wäre. Das empfand auch der Komm. [entator], nur macht er es sich noch leichter als der Rez.[ensent]. Es ist doch dem Kontext unschwer abzulesen, daß man sich wegen der Gleichartigkeit, wie Bier in Philosophenhäuser kommt, um die andere Seite kümmern muß, nach dem einfachsten Analogieschlußverfahren der Welt \& ohne Einweihung in geheime Pedantenkünste. Ich habe mich selbst erst darum kümmern müssen, wie das Bier in Hegels Haus kam ohne auf Trunksucht auszugreifen, weil das den Witz des Satzes zerstörte ${ }^{16}$.

Der Rezensent hatte sich Blumenbergs Zorn durch seinen Mangel an Logik und hermeneutischem Sinn zugezogen. Gründe für seine Bemerkung, dieser habe die Voraussetzungen für eine solche Rezension nicht besessen, ließen sich auch darüber hinaus finden. Auffällig ist, dass es in dieser Besprechung der Bloch'schen Briefe überhaupt nicht um Philosophie, also um Blochs Werk, ging. Am Ende macht sich der Rezensent, der über Blochs anhaltende Geldnot und Schnorrerei gespottet hatte, auch noch persönlich angreifbar, indem er bekennt, ,daß ich mir eine Zeitlang in seinem Hause mein Stipendium aufbessern konnte, von ihm bei gelegentlichen Begegnungen freundlich und so ganz und gar nicht herablassend aufgenommen"17.

Für Blumenberg erwächst aus der laxen Antwort Unselds ein neuer Konflikt; er sieht sich, ,auf dem Tiefpunkt des Werturteils angekommen, wenn in einem Brief des eigenen Verlegers steht: Wer kennt schon die

15. - Gemeint ist der in Australien lehrende Literaturwissenschaftler Gerhard Schulz, dessen denkerische Qualitäten Blumenberg hier in Zweifel zieht.

16. - Da Hans Blumenberg den Text auf seiner eigenen Maschine tippte, enthält er neben den Abkürzungen eine größere Anzahl an offensichtlichen Tippfehlern, die korrigiert wurden. Das Bier in Hegels Haus erwähnt Blumenberg in einem weiteren Nachlasstext: „Bier bei Hegel“, in dem er auf Schopenhauers berühmtes Diktum von Hegels „Bierwirtsphysiognomie“ eingeht. Er übernimmt den Begriff in der gedruckten Fassung des Textes als Motto.

17. - G. Schulz, „Ein Imperator im Reiche des Geistes...“, a.a.O. 
verwandtschaftlichen Beziehungen der Frauen von Philosophen? War etwa gemeint: Und vorwiegend diese oder derartig Belangloses" $" 18$ ?

Vermutlich ist der ausformulierte, aber nicht ausgeschriebene Essay unmittelbarer Ausdruck des Ärgers; eine Verarbeitung in einem Brief oder anderen Text ist jedenfalls nicht überliefert. Im Autor-VerlegerBriefwechsel tritt eine Pause von zwei Monaten ein. Der Leserbrief erscheint, wie gesagt, erst posthum in der Reihe Suhrkamp Taschenbuch Wissenschaft (stw 1755). Die Auseinandersetzung und die damit verbundene Beschäftigung mit dem Nürnberger Haus Tucher weiß der Philosoph aber am Ende doch noch ins Positive zu wenden:

So kam ich dazu, mich um Frau Hegel, ihren Briefwechsel mit ihrer Mutter zu kümmern - und dabei auf e.[ine] Information zu geraten, die meinem nächsten Buch nützlich sein wird \& mich wiederum in den Verdacht des Pedanten setzt: Ich weiß jetzt, wie Hegel an seine Inf. [ormationen] über C.[aspar] H.[auser] kam. Und das ist unentbehrlich f[ür] d[ie] „Höhlenausgänge“ 19 !

\section{Götterlieblinge}

Zwei Jahre später kommt es erneut zu einer Begegnung mit Marcel Reich-Ranicki auf dem philologischen Feld. Die Bedeutung des Konflikts ist hier ungleich größer, geht es dabei doch um einen für alle Beteiligten enorm wichtigen Autor: um Johann Wolfgang Goethe.

Der Konflikt entspinnt sich wohl nicht zufällig mit bzw. über Siegfried Unseld als großen Goethe-Verehrer ${ }^{20}$ und als Blumenbergs Verleger und Gesprächspartner.

Als Hausautor erhielt Hans Blumenberg nicht nur eine ganze Reihe an Publikationen wunsch- und geschenkweise vom Verlag, sondern auch die jeweilige Jahres- bzw. Halbjahres-Vorschau. In einem ausgesprochen kenntnisreichen Verlagstext kündigte der Insel Verlag (der seit 1963 zum Suhrkamp Verlag gehört) im ersten Halbjahr 1987 im Rahmen des Jubiläumsprogramms zum 75. Gründungstag des Verlags einen Band Liebesgedichte Goethes mit Interpretationen bekannter Persönlichkeiten an, herausgegeben von Marcel Reich-Ranicki21. Dieser Band der

18. - Nachlass Hans Blumenberg, DLA Marbach.

19. - Kaspar Hauser (1812-1833) hatte einige Zeit bei seinem Vormund, Gottlieb von Tucher, dem Bruder von Hegels Frau Marie, und zeitweise auch bei deren Mutter, Susanna von Tucher, gewohnt. Zu Kaspar Hauser: Hans Blumenberg, Höhlenausgänge, Frankfurt a. M., Suhrkamp-Verlag, 1989, S. 399f.

20. - Siegfried Unseld veröffentlichte mehrere Bücher über Goethe bzw. schrieb Vorworte zu Editionen.

21. - Alle Freuden, die unendlichen. Liebesgedichte und Interpretationen, hrsg. von Marcel Reich-Ranicki, Frankfurt a. M., Insel-Verlag, 1987. 
Insel-Bücherei mit der Nummer 1028 war Teil der Jubiläumskassette, die Siegfried Unseld dem Philosophen am 6. Mai 1987 zukommen ließ, vermutlich um das besondere Verhältnis Blumenbergs zu der Reihe wissend. In seinem Brief vom folgenden Tag machte ihn Unseld mehr als deutlich auf zwei eigene Texte darin aufmerksam: „Peinlich ist mir nur, daß sich darin wieder zwei Texte von mir befinden und Sie sich genötigt sehen, meinen Fehlern nachzuspüren. Der eine Text befindet sich im Bande der ,Liebesgedichte' von Goethe und der andere in der Bibliographie“ 22 .

Erst Wochen später, am 29. Juni 1987, bedankte sich Hans Blumenberg für die Jubiläumskassette und erinnerte sich an frühe Leseerfahrungen mit dieser Reihe. Er lobt Unselds Interpretation von Goethes Gedicht „Gegenwart", die zuerst in der FAZ vom 24. Mai 1986 erschienen war und in die Goethe-Gedichtauswahl übernommen wurde 23 . Er ist ,entzückt von der Nähe zum Gedicht und seinem Ursprung" 24 und erlaubt sich im gleichen Atemzug einen Seitenhieb gegen den Wiederdruck von Walter Jens' wenig gelungener Interpretation von Goethes „Vor Gericht“ 25 im gleichen Band, ohne jedoch dessen Namen und den des Herausgebers Reich-Ranicki zu nennen. Mit Blick auf Marcel Reich-Ranicki heißt es im Anschluss: „Man druckt das nun wieder, oder sollte man sagen: drückt es durch, weil man der Furcht aller des Wortes Fähigen sicher ist" 26 .

Den bereits erwähnten Ankündigungstext hebt Blumenberg anerkennend hervor, um jedoch im selben Atemzug die im Gedichtband verwendete Variante eines Goethe-Gedichts zu kritisieren:

Als das Insel-Programm 1987/I mit der Ankündigung der GoetheLiebeslyrik vor mir lag, las ich aus dem Auguste-Stolberg-Brief den Vierzeiler, der Titel und Motto des Bändchens aufs schönste hergegeben hat. Ich las aufatmend, denn der Text war richtig! „Alles gaben

22. - Brief von Siegfried Unseld an Hans Blumenberg vom 7.5.1987, Nachlass Hans Blumenberg, DLA Marbach. Dem Brief liegt ein Lieferschein vom 6. 5. bei. Die von dem ostdeutschen Mathematiker und Bibliophilen Herbert Kästner erstellte Verzeichnis 75 Jahre Insel Bücherei 1912-1987. Eine Bibliographie erschien sowohl in der DDR als auch in der 25-bändigen Jubiläumskassette des Insel-Verlags in der BRD. Das Vorwort des Herausgebers wurde jedoch in der Frankfurter Ausgabe durch eines von Siegfried Unseld ersetzt.

23. - Siegfried Unseld, „Gelegenheit“, in: Alle Freuden, die unendlichen, a.a.O., S. 94-96. Der Zeitungsausschnitt befindet sich in Blumenbergs Nachlass.

24. - Brief von Hans Blumenberg an Siegfried Unseld vom 29.6.1987, Nachlass Hans Blumenberg, DLA Marbach.

25. - Walter Jens, „Die Beschuldigte als Richterin“, in: Alle Freuden, die unendlichen, a.a.O., S. 40-42.

26. - Brief von Hans Blumenberg an Siegfried Unseld vom 29.6.1987, Nachlass Hans Blumenberg, DLA Marbach. 
Götter die unendlichen..." ${ }^{27}$. So hatten wir es nicht ${ }^{28}$ gelernt, und so stand es nicht in den Ausgaben unserer Lebenszeit. Immer war es das hochgemute Präsens, und immer stand das Komma da (dem man die Erleichterung für den Leser auch nicht abnehmen möchte). Aber schon Leopold Stolberg hat, kaum drei Jahre daß der Brief geschrieben war, die Verlesung in die Welt gesetzt. Von der Speck-Collection in Yale kann man sich den Film des Autographs kommen lassen und die Vergangenheitsform nachlesen. Goethe wußte, weshalb er sie gebrauchte (darüber anderwärts). Aber nun in Widerruf des Programms, steht das falsche und fälschlich geliebte Präsens doch wieder dem Buch voran. Um die Liebhaber der Insel-Bücherei nicht zu enttäuschen? Oder weil auch hier Marcellus non papa ex cathedra zu entscheiden hatte, was gilt $^{29}$ ?

Die ironische Entthronung des „Literaturpapstes“30 bei gleichzeitiger Kritik an dessen gerade nicht unfehlbaren Urteilen wird dem Philosophen in seiner Mischung aus kirchen-, musik- und literaturkritischen Bezugnahmen Vergnügen bereitet haben ${ }^{31}$.

Das titelgebende Gedicht ist als Motto gleich anfangs abgedruckt; den Kommentar dazu liefert erst das Nachwort (,Statt eines Nachworts“) des Herausgebers Marcel Reich-Ranicki.

Um den Konflikt und das dahinterstehende interpretatorische Problem verständlich zu machen, soll die wechselvolle Geschichte des Gedichts hier kurz erläutert werden. Johann Wolfgang Goethe stand mit der vier Jahre jüngeren Augusta Louise zu Stolberg (1753-1835) jahrelang in Briefwechsel, ohne „Gustgen“ je gesehen zu haben. Am 17. Juli 1777 schrieb er ihr einen Brief, in dem es um den Tod seiner Schwester Cornelia (1750-1777) geht. Darin findet sich ein kurzes und sehr persönliches Gelegenheitsgedicht, das die Gefühle nach dem Tod seiner Schwester wiederspiegelt. ${ }^{32}$ Dieses Gedicht, das Goethe selbst nie hat drucken lassen, zählt heute zu seinen bekanntesten Gedichten:

27. - Hervorhebung von Hans Blumenberg.

28. - Kursivierung des „nicht“ als Hervorhebung der Verfasserin D.K.

29. - Brief von Hans Blumenberg an Siegfried Unseld vom 29.6.1987, Nachlass Hans Blumenberg, DLA Marbach.

30. - In kritischer Absicht hatte Martin Walser dem Kritiker in seinem Artikel „Über Päpste. Von Kritikern, die im Besitz eines absoluten Wissens sind“ in der Zeit vom 25.3.1977 ungenannt den Titel des Literaturpapstes verliehen. Dort heißt es u.a.: „Die katholische Kirche hat viel, viel länger gebraucht, ihren Päpsten ein Unfehlbarkeitsdogma zu erwirken, als das bürgerliche Feuilleton.“

31. - Blumenberg hat hier den Renaissance-Komponisten Jakobus Clemens non papa mit Palestrinas bekannter Papst-Krönungsmesse „Missa papae Marcelli“ von 1562 zusammengeführt.

32. - Faksimile des Briefs vom 17.7.1777, in: Carl Frederick Schreiber, Goethe's works with the Exception of Faust. A Catalogue compiled by the members of Yale University Library Staff, New Haven 1940, nach S. 4, Nr. 526. 
Alles gaben Götter die unendlichen
Ihren Lieblingen ganz
Alle Freuden die unendlichen
Alle Schmerzen die unendlichen ganz.

Es wurde drei Jahre nach Erhalt des Briefs von Augustas Bruder Friedrich (Fritz) Leopold zu Stolberg, einem Freund Goethes, im Deutschen Museum erstmals veröffentlicht ${ }^{33}$. Und während die handschriftliche Version Goethes keine Kommas und nur einen einzigen Punkt am Ende kennt, fanden sich im Druck drei Kommas und zwei Ausrufezeichen im Text, vermehrt um etliche Veränderungen in der Groß- und Kleinschreibung. Zudem hatte Fritz zu Stolberg in der ersten Zeile einen Artikel eingefügt und das Tempus verändert. Aus: „Alles gaben Götter die unendlichen...“ wurde nun „Alles geben die Götter, die Unendlichen...34“.

In den später gedruckten Ausgaben wurde fast durchgängig Stolbergs Version nachgedruckt. Der Wechsel des Tempus verändert jedoch den Sinn des Gedichts wesentlich: Hatte Goethe die freudvollen und leidvollen Gaben der Götter im persönlichen Schmerz um die Schwester ins Präteritum, eine Vergangenheitsform, gesetzt, so wurden sie nun durch das Präsens in der Stolberg-Variante generalisiert und entindividualisiert. Vermutlich hat gerade diese Verallgemeinerung dem Gedicht den Weg zu so großer Bekanntheit geebnet. Von Goethe selbst ist keine spätere Äußerung zum Gedicht überliefert. In den Werkausgaben existieren die verschiedensten Schreibungs- und Zeichensetzungsvarianten; der eingefügte Artikel und die Gegenwartsform finden sich jedoch bis in die neuere Zeit fast durchgängig. Dabei lag der handschriftliche Brief seit 1940 als Faksimile gedruckt vor ${ }^{35}$. Der von Marcel Reich-Ranicki herausgegebene Band gibt das Gedicht in einer der Stolberg'schen sehr ähnlichen Variante wieder ${ }^{36}$. Die Kommas sind geblieben, Ausrufezeichen und Großschreibungen zurückgenommen. Beibehalten wurde jedoch der hinzugefügte Artikel und die veränderte Zeitform mit dem Zusatz „Goethe im Brief an die Gräfin Auguste zu Stolberg“. Dem aber entstammte die wiedergegebene Form ganz sicher nicht.

33. - Friedrich Leopold zu Stolberg, „Ueber die Ruhe nach dem Genuß und über den Zustand des Dichters in dieser Ruhe", in: Deutsches Museum, Bd. 2, Leipzig 1780, 7. Stück, S. 7.

34. - Vgl. die Varianten bei Schreiber bzw. im Deutschen Museum, a.a.O.

35. - Carl Frederick Schreiber, Goethe's works with the Exception of Faust..., a.a.O.

36. - In den bibliographischen Notizen wird die Ausgabe: Goethes Gedichte in zeitlicher Folge, hrsg. von Heinz Nicolai, Frankfurt a. M., Insel-Verlag, 1982 genannt. Dort findet sich das Gedicht auf S. 216. 
Will man sich den Unmut des Philosophen erklären, kann man in einem seiner nachgelassenen Goethe-Essays Generelles über die Bedeutung wissenschaftlicher Präzision lesen:

Genauigkeit ist ein wissenschaftliches Ideal, und immer wieder hat sich gezeigt, daß Steigerung der Anforderung an Genauigkeit identisch ist mit dem Sichtbarwerden neuer Phänomene und daher mit der Nötigung zu theoretischen Revisionen. / Die technisch-wissenschaftliche Zivilisation hat Genauigkeit auch zu einem Stück unserer Lebensmoral gemacht. Da kann sie identisch werden mit Unerbittlichkeit ${ }^{37}$.

Siegfried Unseld, selbst promovierter Germanist, bemühte sich um Schadensbegrenzung, indem er die Bedeutung des aufflammenden Konflikts zwischen seinen Hausautoren herunterspielte: „Ich muß bekennen, ich kannte die Differenzen gar nicht und ich kenne diese vier Zeilen auswendig, leider im Präsens"38. Für die zweite Auflage versprach er den Austausch des falschen Textes ${ }^{39}$. Das „Monitum“ gab er an Marcel Reich-Ranicki weiter.

Dieser antwortete Blumenberg am 6. Juli 1987 in aller Kürze: „Ich fürchte sehr, daß Sie mein Nachwort zu diesem kleinen Band nicht gelesen haben. In diesem Nachwort habe ich versucht zu erklären, warum ich mich für diese Fassung entscheide" 40 .

Reich-Ranicki thematisiert in seinem Text „Statt eines Nachworts“ tatsächlich die unterschiedlichen Varianten des Gedichts ausführlich, zitiert sogar den Anfang der im Brief überlieferten Form. In Bezug auf die beiden entscheidenden Veränderungen fragt er:

Hat also Stolberg eigenmächtig gehandelt? Goethe kannte die Zeitschrift „Deutsches Museum“ und erwähnte sie gelegentlich. Daher ist der Gedanke nicht abwegig, daß er auch den Aufsatz mit dem zitierten Gedicht gelesen und die eventuell von Stolberg vorgenommenen Änderungen gebilligt hat - stillschweigend oder vielleicht in einem verlorengegangenen Brief $^{41}$.

Aber welche Begründung für die Auswahl der von ihm ausgewählten Form gibt Reich-Ranicki an?

37. - Hans Blumenberg, Goethe zum Beispiel, in Verbindung mit Manfred Sommer hrsg. vom Hans-Blumenberg-Archiv, Frankfurt a. M. und Leipzig, InselVerlag, 1999, S. 83f., hier S. 83.

38. - Brief von Siegfried Unseld an Hans Blumenberg vom 7.7.1987, Nachlass Hans Blumenberg, DLA Marbach. Ein Tippfehler (Präsenz anstelle von Präsens) wurde korrigiert.

39. - Im Jahr 2005 erschien die 7. Auflage des Buches in unveränderter Form.

40. - Nachlass Hans Blumenberg, DLA Marbach.

41. - Marcel Reich-Ranicki, „Statt eines Nachworts“, in: Alle Freuden, die unendlichen, a.a.O., S. 171-176, hier S. 173. 
Es läßt sich doch nicht verheimlichen, daß Stolbergs Version der ursprünglichen Fassung entschieden vorzuziehen ist. Erstens: Die Hinzufügung des Artikels „die“ verleiht auch dem ersten Vers den gleichmäßigen Rhythmus der drei übrigen Verse. Und nichts spricht dafür, dass Goethe daran gelegen war, diesen Rhythmus zunächst zu durchbrechen. Zweitens: Von der Zeit, in der das Verbum ,geben“ verwendet wird, hängt der Sinn des ganzen Vierzeilers ab. Der Dichter, der ,gaben“ schreibt, drückt eine allgemeine Erfahrung und eine geschichtliche Erkenntnis aus: So war es, behauptet er, einst, vielleicht in grauer Vorzeit. Wenn er aber ,geben“ sagt, dann formuliert er einen keineswegs zurückliegenden, vielmehr gegenwärtigen Zustand und damit (möglicherweise) eine persönliche Erfahrung. Was wollte Goethe ${ }^{42}$ ?

Diese interessante Frage beantwortete der Autor des Gedichts insofern, als er das Gedicht im Brief als solches stehen ließ, es in keinen Gedichtband aufnahm und sich nie dazu geäußert hat. Als ambitionierter Philologe erweist sich Marcel Reich-Ranicki mit seinen phantasievollen Begründungen ganz sicher nicht, aber wie in vielen anderen Fällen als Sachwalter des Publikumsgeschmacks, dessen Legitimität er verteidigt. Und vermutlich fühlte er sich im Recht, hatte er sich doch nicht an einer kritischen Edition versucht, sondern eine Auswahl an GoetheGedichten mit Interpretationen herausgegeben, die alle - abgesehen von seiner eigenen Interpretation im Nachwort - bereits in der Frankfurter Anthologie erschienen waren.

Hans Blumenberg muss die lapidare Antwort Reich-Ranickis getroffen haben. In seinem Nachlass findet sich der Entwurf zu einem Brief an ihn, von dem man annehmen kann, dass er nie abgeschickt wurde $^{43}$. Er beginnt polemisch und kommt schnell zur Sache:

Jedermann weiß, daß es unmöglich ist, vor dem Richtstuhl des Geschmacks recht zu behalten oder Recht zu bekommen. / Mal versäumt man die Frist, wie es mir mal mit dem biertrunkenen Hegel erging (genehmere Fälle sind auch bei Fristversäumnis noch tolerierbar), mal hat man die Akten nicht ausreichend studiert, wie im vorliegenden Fall des [wein?]trunkenen Goethe ${ }^{44}$.

Der Entwurf thematisiert auch den Widerspruch zwischen der in der Verlagsankündigung wiedergegebenen richtigen Form des Gedichts

42. - Ebd., S. 174.

43. - In Marcel Reich-Ranickis sehr konzentriertem Nachlass im DLA Marbach existiert der Brief nicht. Der hier zitierte „Entwurf 10.7.87 an MRR“ findet sich in Hans Blumenbergs Nachlass im DLA Marbach.

44. - Der erste Teil des Worts „weintrunken“ war nicht sicher zu entziffern und steht deshalb in Klammern. In einer gestrichenen Variante ist zu lesen: „...wie im vorliegenden Goethefall, dem eines ganz untrunkenen.“ 
und der gedruckten Version. Erst am Ende formuliert Blumenberg die inhaltlichen Einwände, die er im Brief an Unseld (,darüber anderwärts“) ausgespart hatte.

\begin{abstract}
Nun, nach der Lektüre Ihres Nicht-Nachwortes, muß ich nochmals geständig werden: Es freut mich, die mir bekannten Gründe für die geläufigere, vertrautere Fassung vermehrt zu sehen. Daß Sie gegenläufige Argumente nicht abwägen, ist dem Kritiker - dessen Geschäft ich seit 30 Jahren nur als Zuschauer kenne - wohl erlaubt. Als NichtKritiker ${ }^{45}$ werde ich das, was ich zur Sache schon geschrieben habe, neuerdings umwälzen. Das „lyrische Ich“ kann in einem Brief nicht den Pardon für jederlei Kühnheit abgeben - und dieses ist, obwohl in der Situation versetzt, eben doch vor allem einem Brieftext integriert. Ich bin überzeugt, daß G.[oethe] bei der Hereinnahme in e[ine] Werkausgabe $n$ [icht] hätte drucken lassen, wie Sie es wünschen - und ich auch. Aber Wünsche gelten nun einmal für $\mathrm{H}[\mathrm{and}] \mathrm{s}$ [chriften] nicht (G. schrieb damals noch eige[n]h[än]d[i]g-) ${ }^{46}$.
\end{abstract}

Blumenberg gesteht - wie im Brief an Unseld - eine gewisse Affinität zur „falschen und fälschlich geliebten“ Textfassung seiner Kinderzeit ein, verweist aber auf die von Goethe niemals aufgehobene Verankerung in den Brieftext. In der handschriftlichen Randbemerkung rekurriert er auf den pluralischen Gottesbegriff im Gedicht, verweist indirekt auf seine Ausführungen zum Promethismus und zu den Polytheismen bei Goethe in seinem 1979 erschienenen Buch Arbeit am Mythos. Hier hatte er auch Goethes bekanntes „Apophthegma“ zitiert: „Wir sind naturforschend Pantheisten, dichtend Polytheisten, sittlich Monotheisten“47. Sein Kommentar zu Goethes „ungeheurem Spruch“ („Nemo contra deum nisi deus ipse“) ließe sich wohl auch auf seine Position zum umstrittenen Gedicht übertragen: „Dabei ist die sprachliche Gestalt nicht kontingent, nicht äußere Zutat, sondern mit der immanenten Genese eng verknüpft“"48.

Die emotionale Bedeutung, die Goethe als Vertreter eines humanistischen Bildungsideals und einer klassischen Ästhetik für die Söhne aus bürgerlichem Hause lebenslang hatte, ist nicht zu unterschätzen,

45. - Hier eingefügt, ohne den weiterlaufenden Text gestrichen zu haben, ist folgender Einschub: Als Nicht-Kritiker „kann ich mir nicht herausnehmen, die zu G[oethe]s Gedicht zu stellenden Fragen auch hier zu st[ellen]: wer spricht wann? G[oethe]s Zimperlichkeit im Umgang mit s.[einen] Polytheismen u. [nd] s.[einem] Prom[ethismus]!“ Die Lesart der letzten Phrase ist unsicher. D.K.

46. - „Entwurf 10.7.87 an MRR“, Nachlass Hans Blumenberg, DLA Marbach.

47. - Hans Blumenberg, Arbeit am Mythos, Frankfurt a. M., Suhrkamp-Verlag, 1979, S. 585. Als Quelle führt Blumenberg Goethes Maximen und Reflexionen, Werke IX, S. 745 an. Goethe hatte den Satz auf einen Briefentwurf an Friedrich Heinrich Jacobi vom 6.1.1813 geschrieben.

48. - Ebd., S. 587. 
verbanden sich doch für beide bzw. für alle drei (Siegfried Unseld eingeschlossen) damit Erinnerungen an eine prägende Schulzeit vor der Machtübernahme der Nationalsozialisten. Aus diesem Vorrat speiste sich das identifikatorische Potential, das sich mit Goethes Namen verband und der Auseinandersetzung eine sehr persönliche Note verlieh. Im Gedicht über die freudvollen und leidvollen Göttergaben hatte Marcel Reich-Ranicki eine „lapidare Selbstcharakteristik Goethes“erkannt und hinzugefügt: ,Aber es betrifft nicht nur ihn selber“49. Nein, es betraf auch ihn, den jüdischen Provokateur einer deutschen Öffentlichkeit, zu der er gehörte und der er immer wieder - zuletzt 2008 bei der Festgala zur Verleihung des deutschen Fernsehpreises - den Spiegel vorhielt. Über Goethe schreibt er:

Er war unter allen großen Deutschen der menschlichste - menschlich in seiner Sehnsucht nach Liebe und Frauen, menschlich in seiner Angst vor Kritik, menschlich in seinem Bedürfnis nach Beifall, in seiner Empfänglichkeit für Lob und in seiner Abhängigkeit vom Erfolg ${ }^{50}$.

Hans Blumenberg näherte sich Goethe auf distanziertere Weise ${ }^{51}$. Der zeitliche Abstand zu dem so bewunderten wie kritisierten Autor ermöglichte es ihm - gerade über die autobiographischen Schriften und die Zeugnisse aus Goethes Umfeld vermittelt - zum nacherlebenden Zuschauer zu werden und Lebensprobleme, auch die eigenen, aus einer wohltuenden „Distanz zu sich selbst" 52 zu betrachten. Er zitiert Nietzsche, der im Blick auf das eigene Leben empfohlen hatte, wie der Betrachter eines Bildes Abstand zu nehmen und „nicht zu genau sehen zu wollen", wie Goethe es verstanden habe. Beispielhaft steht Goethe hier für ein Problem, ,das wir alle mit uns selbst haben: unsere Optik auf uns so einzustellen, dass sie uns weder demütigt noch bläht. Man muss ertragen können: die Welt, die anderen, das Andere (wozu der Schmerz und das Leid gehören) - vor allem: sich selbst" 53 . Am Beispiel Goethes widmet sich Blumenberg den Problemen des Alterns und des Sterbens, der Einsamkeit und des Vergessenwerdens. Unter der

49. - M. Reich-Ranicki, Alle Freuden, die unendlichen ..., a.a.O., S. 175.

50. - Marcel Reich-Ranicki, „Der Verächter der Kritik“, in: Ders., Goethe noch einmal. Reden und Anmerkungen, Stuttgart, München, DVA, 2002, S. 57-75, hier S. 73. Goethes Gedicht „Der Rezensent“, das mit den Worten endet: „Schlagt ihn tot, den Hund! Er ist ein Rezensent.“ nennt Reich-Ranicki ,das dümmste, das aus seiner Feder stammt." FAZ vom 20.1.1990 und in Band 14 der Frankfurter Anthologie, Frankfurt a. M., Insel, 1991, S. 29-32.

51. - Die folgenden Zitate stammen aus dem von Hans Blumenberg geplanten, aber erst aus dem Nachlass edierten Buch: Hans Blumenberg, Goethe zum Beispiel, Frankfurt a. M., Suhrkamp, 1999.

52. - Ebd., S. 83.

53. - Ebd., S. 83f. 
„Erkaltung des Zeitgeistes“, dieses „launischen Dämons“, habe gerade derjenige zu leiden, der „den Beifall der Zeitgenossenschaft einmal gekostet hat, dieses süße Gift für den Aufbau des Selbstbewußtseins aus eigenen Mitteln und an eigenen Maßstäben [... ${ }^{\text {“554. }} \mathrm{Zu}$ denen aber gehörten sowohl Hans Blumenberg als auch Marcel Reich-Ranicki, die unter den „Zumutungen des Zeitgeistes“ 55 gelitten hatten, aber keineswegs vergessen waren oder sind.

Der Auseinandersetzung zwischen beiden folgte noch ein kurzes Nachspiel, bei dem man fast meinen könnte, der Kritiker hätte den verstorbenen Philosophen narren wollen. Im August 1999, drei Jahre nach Blumenbergs Tod, veröffentlichte Marcel Reich-Ranicki noch einmal eine Variante seines "Nachworts" in der FAZ im Rahmen der Frankfurter Anthologie ${ }^{56}$. Der gekürzte Text beginnt mit dem Bekenntnis: „Ich liebe dieses Gedicht, diese achtzehn Worte. Hier stocke ich schon. Sind es wirklich achtzehn Worte oder vielleicht nur siebzehn? Goethe selber hat den vier Versen - man kann es kaum glauben - keine Bedeutung beigemessen." Einiges von der Schärfe seiner Urteile ist zurückgenommen, der Grundtenor jedoch geblieben. Das so geliebte Gedicht hat erst in der "hundert Jahre lang in allen Goethe-Ausgaben“ gedruckten Form seine Vollendung erreicht. In der Fassung von eigener Hand hatte der Autor ein unpassendes Tempus gewählt, einen Artikel und zwei Kommas vergessen. Goethe, der aus einer Weinhändlerfamilie stammte und - ,der gern trank“, - hätte darüber vermutlich gelächelt.

54. - Ebd., S. 200.

55. - Ebd., S. 199.

56. - „Alles geben die Götter“" von Johann Wolfgang von Goethe, $F A Z$ vom 28.8.1999, nochmals im 23. Band der Frankfurter Anthologie, hrsg. von Marcel ReichRanicki, Frankfurt a.M., Insel-Verlag, 2000, S. 53ff. 